\title{
Adaptabilidad eco-territorial del cultivo de tomate para la producción agrícola sostenible en Carabobo, Venezuela
}

\author{
Eco-territorial adaptability of tomato crops for sustainable \\ agricultural production in Carabobo, Venezuela \\ Barlin Orlando Olivares ${ }^{1 *}$, Rafael Hernández ${ }^{2}$, Alexander Arias ${ }^{2}$, \\ Juan Carlos Molina' ${ }^{2}$ Yessica Pereira ${ }^{2}$
}

\section{RESUMEN}

La zonificación genera mejores elementos de decisión para el manejo de los cultivos y conduce a una racionalización del uso de recursos de cualquier territorio. Por lo anterior, el objetivo de este trabajo fue definir las áreas idóneas para el cultivo del tomate desde el punto de vista agroecológico, en el estado Carabobo, Venezuela. Se utilizaron datos diarios de precipitación de 41 estaciones climáticas, cartas edafológicas de la entidad y programas de cómputo para los balances hídricos del cultivo para estimar los rendimientos potenciales de acuerdo con la fecha de siembra. El análisis de las condiciones climáticas y edáficas de las tierras agrícolas del estado indica que se tienen $348 \mathrm{~km}^{2}$ de suelos apropiados para el cultivo de tomate, las tierras se ubican en zonas de llanura lacustrina, valles, colinas y montañas con suelos de textura franca y moderadamente drenados, pudiendo alcanzar valores del $100 \%$ de rendimiento en cosecha.

Palabras clave: Agricultura, climatología, regionalización, sustentabilidad, territorio.

\begin{abstract}
The zoning generates better decision elements for the management of crops and leads to a rationalization in the use of the resources of any territory. Therefore, the objective of this work was to define the ideal areas for the cultivation of tomatoes from the agroecological point of view in the state of Carabobo, Venezuela. Daily rainfall data were used from 41 climatic stations, edaphological charts of the entity and computer programs for the water balance of the crop to estimate potential yields according to the sowing date. The analysis of the climatic and edaphic conditions of the agricultural lands of the state, indicates that there are $348 \mathrm{~km}^{2}$ of suitable soils for tomato cultivation, the lands are located in zones of lacustrine plain, valleys, hills and mountains with soils of frank texture and moderately drained, being able to reach values of $100 \%$ of the harvest yield.
\end{abstract}

Keywords: Agriculture, climatology, regionalization, sustainability, territory.

\section{Introducción}

Venezuela cuenta con una gran variabilidad de suelos, recursos hídricos y tipos de clima en las diferentes regiones naturales, lo cual plantea una oportunidad para planificar el uso y manejo, de acuerdo con una zonificación agrícola basada en las limitaciones y potencialidades de las tierras. El fortalecimiento de la producción de hortalizas mediante el uso eficiente de los recursos naturales para incrementar la productividad, es esencial para lograr el ingreso de divisas y garantizar el abasto nacional; por lo que los estudios de zonificación de cultivos juegan un papel importante para lograr estas metas (Villa et al., 2001).

El tomate (Solanum lycopersicum) en Venezuela se siembra en el occidente, por sus características físico-naturales y por tradición (INIA 2016). La producción es destinada al consumo inmediato, por lo que aproximadamente el $80 \%$ se traslada a los mercados de mayoristas locales y regionales, mientras que el resto se utiliza en la agroindustria para el procesamiento de pasta de tomate (Nava et al., 2013).

1 Campus de Excelencia Internacional Agroalimentario (ceiA3). Programa Iberoamericano de Doctores en Agroalimentación. Universidad de Córdoba (UCO), España.

2 Gerencia de Meteorología Aplicada, Instituto Nacional de Meteorología e Hidrología (INAMEH), Venezuela.

* Autor de correspondencia: barlinolivares@gmail.com; ep2olcab@uco.es. 
El rendimiento del tomate es bajo en la mayoría de los países tropicales, debido al efecto negativo que ejercen los factores climáticos, como las altas temperaturas, lluvias y humedad relativa (Cortez et al., 2016), así como la incidencia de plagas y enfermedades (Solís et al., 2006), y las condiciones adversas como sequías (Olivares et al., 2016; Olivares y Zingaretti, 2018). En Carabobo, el tomate es uno de los principales cultivos tradicionales caracterizado mayormente por paquetes tecnológicos convencionales y cuya expansión en varias zonas agrícolas se ha hecho más evidente en los últimos años. Esto ha generado el interés de mejorar los sistemas productivos debido al decrecimiento significativo, alcanzando mínimos históricos en el período 2008-2015, donde el cultivo de tomate obtuvo una cifra de $-18,7 \%$ de la producción caída (IANAS, 2017). Es precisamente por esta razón que el desarrollo de una adecuada zonificación agroecológica en la región bajo estudio, además de posibilitar la localización específica de las áreas con mayor vocación para actividades productivas (Olivares et al., 2018d), permite reconocer la ubicación de la infraestructura disponible para el impulso de la soberanía y seguridad alimentaria del país (Olivares et al., 2018c).

La aptitud de las tierras para diferentes usos puede ser descrita de formas sistemáticas, por lo que los usuarios de tierras, planificadores ambientales, y los servicios agrícolas pueden usar estas predicciones para guiar sus decisiones (Salas et al., 2008). Por lo anterior el objetivo del estudio fue definir áreas idóneas para el cultivo del tomate desde el punto de vista agroecológico en el estado Carabobo, Venezuela.

\section{Materiales y métodos}

\section{Área de estudio}

El estado Carabobo se localiza en la zona centro-norte de Venezuela, entre las coordenadas geográficas $09^{\circ} 48^{\prime} 10^{\prime \prime}$ y $10^{\circ} 35^{\prime} 25^{\prime}$ ' LN y, $67^{\circ} 31$ ' $12^{\prime \prime}$ y $68^{\circ} 26^{\prime} 08^{\prime \prime}$ LO (Figura 1). Está constituido por catorce municipios, tiene una superficie de $4.651 \mathrm{~km}^{2}$ de los cuales el 42,53\% (1.973 $\left.\mathrm{km}^{2}\right)$ cuentan con potencial para la actividad agrícola (Hernandez et al., 2017).

La precipitación promedio anual del estado es de $1.100 \mathrm{~mm}$ (Olivares et al., 2018a). Presenta los siguientes tipos de climas: a) semiárido (D) con lluvia anual inferior a $750 \mathrm{~mm}$, en los municipios Juan José Mora y Puerto Cabello. b) subhúmedo seco (C1) con lluvia anual entre los 920 y $1.060 \mathrm{~mm}$, en los municipios Miranda, Montalbán, Bejuma, Naguanagua, Los Guayos, San Diego, Guácara, San Joaquín y Diego Ibarra; así como el extremo

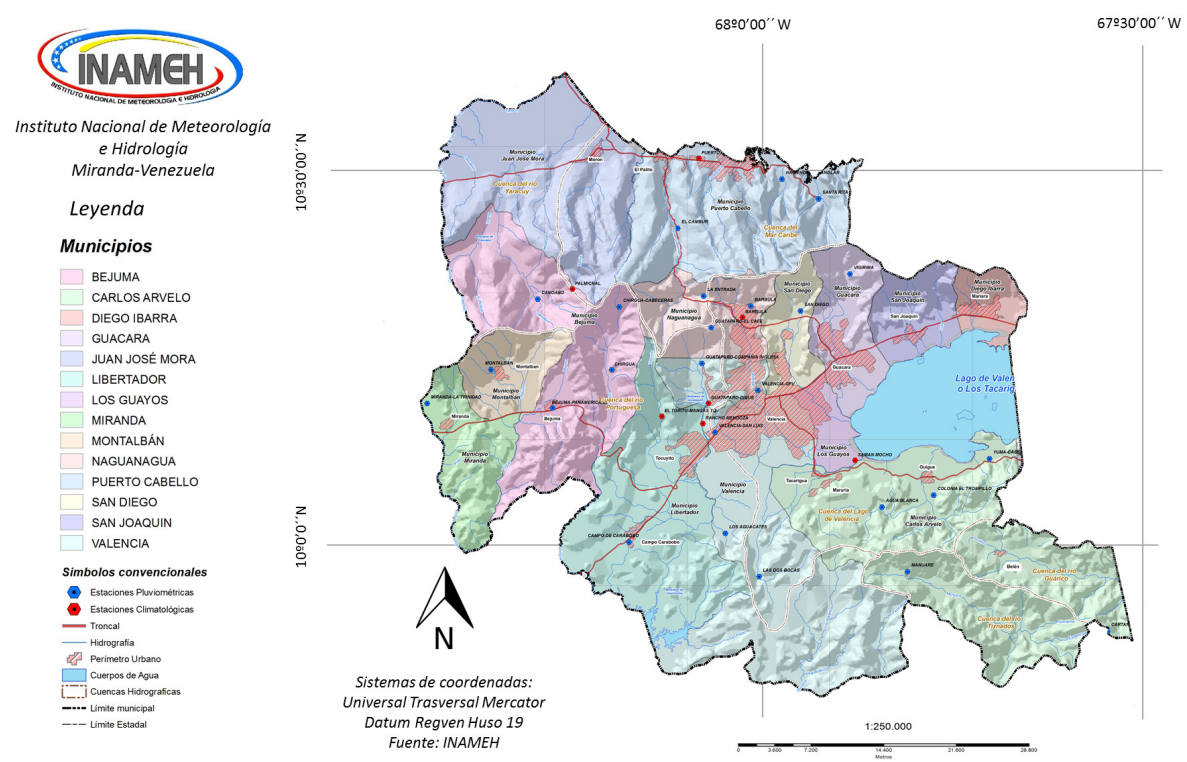

Figura 1. Ubicación geográfica del estado Carabobo, Venezuela. 
norte de los municipios Libertador, Valencia, Carlos Arvelo; y extremo sur de los municipios Juan José de Mora y Puerto Cabello. c) subhúmedo húmedo (C2) con precipitaciones anuales de $1.290 \mathrm{~mm}$, en el área más septentrional del municipio Valencia y meridional del municipio Libertador, así como en el suroeste de Carlos Arvelo, donde se encuentran las áreas agrícolas más extensas y de mayor potencial del estado, y d) ligeramente húmedo (B1) con precipitación anual de $1.540 \mathrm{~mm}$, en el extremo sur del municipio Valencia con los límites del estado Cojedes (Olivares, 2018).

\section{Análisis climático}

Se utilizaron datos de precipitación y evaporación de períodos de diez días de las series (1970-2000) de 41 estaciones meteorológicas ubicadas en los estados Carabobo, Aragua y Yaracuy. Se realizó un control de calidad de los datos de precipitación para definir la proporción de datos faltantes, identificar valores fuera de lo normal y observar la distribución de las series de precipitación (Olivares et al., 2013, Parra y Cortez, 2005). Para determinar las fechas de inicio y duraciones de los períodos de crecimiento y húmedo se utilizó la metodología propuesta por Franquin (1983). Posteriormente, se calcularon los balances hídricos del cultivo para cada estación climatológica según la metodología de la FAO (2012) e implementada por Hernández et al. (2017) y Olivares et al. (2018b), que arrojó información para realizar el análisis de los riesgos edafoclimáticos.

\section{Análisis edáfico}

Se utilizó como base el mapa de clasificación por capacidad de uso de la tierra del estado Carabobo de la Dirección General de Recursos Hidráulicos del Ministerio de Obras Públicas a escala 1:100 000 (Hernández et al., 2017), por ser el único estudio de suelos disponible. Las variables edáficas analizadas fueron el tipo de drenaje, la profundidad, la textura de suelos y la pendiente del terreno, todas indispensables para obtener la capacidad máxima de almacenamiento del suelo (CMA). Adicionalmente, se utilizó el Sistema de Información de las Áreas Agroecológicas (SIAA) del Instituto Nacional de Investigaciones Agrícolas (INIA, 2016) y el Sistema de Información de Suelos de la Depresión del Lago de Valencia (SISDELAV).

\section{Requerimientos del cultivo de tomate}

La definición de los requerimientos del tomate se ovtubo por medio de los aportes de Benacchio (1982) para determinar las necesidades hídricas, temperatura y otros factores climáticos que afectan el cultivo. El tomate, tiene cierta resistencia a la sequía durante el transcurso de sus fases fenológicas, como lo indica Doorenbos y Pruitt (1975), Ortega-Farías et al. (2000) quienes señalan que el coeficiente del cultivo $(\mathrm{Kc})$, aumenta conforme transcurren las décadas de crecimiento del cultivo y después de la fructificación decae. Los valores de coeficiente de cultivo $(\mathrm{Kc})$ se tomaron de Allen et al. (2006).

\section{Zonificación agroecológica}

En este aspecto se compararon los requerimientos del cultivo con la oferta edafoclimática del área, para cuantificar el cumplimiento de estos, y, en consecuencia, que grado de aptitud tiene el área para la siembra del tomate. En primera instancia, se analizaron separadamente las relaciones sueloclima-cultivo, y luego se integraron para obtener un resultado único (MARNR, 1989).

El resultado del balance permitió analizar: la evapotranspiración máxima del cultivo (Etm), el rendimiento del cultivo y el uso del agua (Ky), el coeficiente de uso consuntivo $(\mathrm{Kc})$, la relación entre la demanda de agua del cultivo mantenido a niveles óptimos (ETa) y la demanda del cultivo de referencia (ETP).Además se obtuvo el rendimiento esperado en porcentaje, de sembrarse a una fecha estimada donde se garantice la humedad inicial en los suelos. Todo el conjunto de esta información obtenida de los balances hídricos fue modelado para el mapa de zonificación agroecológica del cultivo de tomate en Carabobo.

La FAO (2012) propone el uso de la ecuación 1 que relaciona la disminución relativa del rendimiento con la disminución relativa de la evapotranspiración (ET). La adaptabilidad de los cultivos al clima del area se determinó sobre la base de los requerimientos hidricos (Eta), mediante la relacion entre la disminucion del rendimiento relativo y el déficit hidrico dado por el factor Ky.

$$
\text { 1-YaYm=Ky 1-ETaETm }
$$

Dónde: (Ya) es el rendimiento real cosechado; (Ym) el rendimiento máximo cosechado; (ky) el 
factor del efecto del déficit sobre el rendimiento; (ETa) la evapotranspiración real del cultivo y (ETm) evapotranspiración máxima del cultivo.

El factor de respuesta del rendimiento (Ky) capta la esencia de las complejas relaciones que existen entre la producción y el uso del agua en un cultivo, donde ocurren procesos biológicos, físicos y químicos.

De acuerdo con la FAO (2012), el procedimiento de cálculo de la ecuación 1 para determinar el rendimiento real consta de los siguientes pasos: I) estimar el máximo rendimiento (Ym) de una variedad adaptada de cultivo, según su comportamiento genético y el clima, suponiendo que los factores agronómicos (agua, fertilizantes, plagas y enfermedades) no son limitantes. II) calcular la evapotranspiración máxima (ETm) según metodologías establecidas y teniendo en cuenta que se han suministrado todos los requerimientos de agua del cultivo. III) determinar la evapotranspiración real (ETa) del cultivo en el caso específico, de acuerdo con el suministro de agua disponible para el cultivo. IV) evaluar el rendimiento real (Ya) mediante la selección apropiada del factor de respuesta (Ky) para todo el período de crecimiento o en cada una de las distintas etapas de crecimiento.

La FAO (2012) recomienda procedimientos para estimar el máximo rendimiento a partir de datos locales disponibles de rendimientos máximos de cultivos o basados en el cálculo de la biomasa máxima y el índice de cosecha correspondiente, mediante el enfoque de zona ecológica. Para luego establecer un criterio general de adaptabilidad de acuerdo con la relación Ya/Ym. Después, para el cálculo de los requerimientos hídricos de los cultivos (Eta) para cada rango de aptitud, tanto para el ciclo vegetativo como para las etapas críticas, se compararon con la oferta climática del área, definida como la precipitación efectiva del $75 \%$ de probabilidad de ocurrencia. Posteriormente, se calculó un índice de adaptabilidad climática total para determinar el grado de aptitud (ecuación 2).

$$
\mathrm{IT}=0.4 * \mathrm{ICV}+0.6 * \mathrm{IFC}
$$

Dónde: (IT) es el Índice total; (ICV) es el Índice del ciclo vegetativo y (IFC) es el Índice de la fase crítica.

La zonificación espacio temporal del cultivo de forma gráfica superponiendo los mapas de aptitud suelo-cultivo y de clima-cultivo se realizó mediante ambiente SIG Arcview v.3.2. considerando como no aptas aquellas zonas que presentaron la característica de no idóneo en cualquiera de los dos mapas de aptitud (suelo-cultivo y clima-cultivo).

\section{Resultados y discusión}

\section{Aptitud de las tierras para el cultivo de tomate}

A título ilustrativo, la Figura 2 muestra la distribución espacial de las categorías de aptitud de las tierras en Carabobo obtenidas mediante el análisis climático y edáfico. La superficie apta

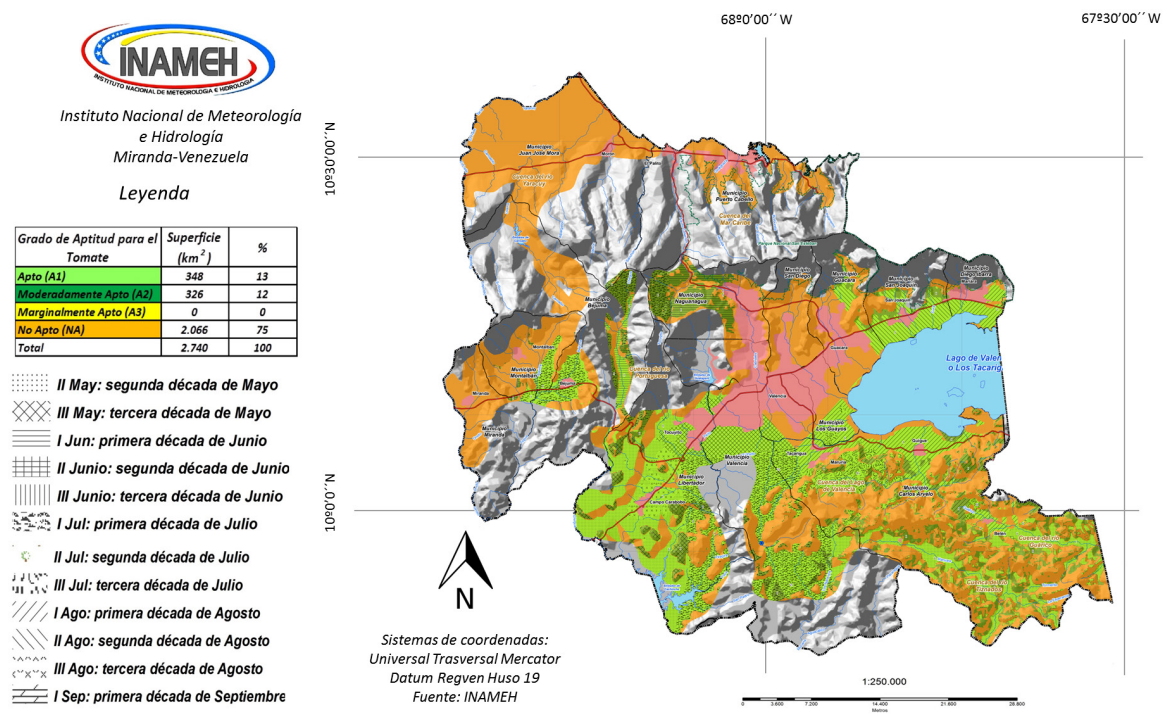

Figura 2. Zonificación agroecológica del cultivo de tomate en Carabobo, Venezuela. 
para el adecuado crecimiento y desarrollo es de $348,0 \mathrm{~km}^{2}$, que equivale al $13,0 \%$ del territorio; mientras que la superficie moderadamente apta fue de $326,0 \mathrm{~km}^{2}$, que corresponde al $12,0 \%$ del estado.

En la Tabla 1 se observa que solamente se tiene $1,0 \mathrm{~km}^{2}$ de suelos aptos para el tomate en el municipio Naguanagua, debido a que las condiciones edafoclimáticas en las áreas bajas de las montañas y colinas de la serranía del litoral, donde se encuentra la mayoría de las tierras cultivables del municipio, no permiten obtener rendimientos mayores del $95,0 \%$, con fecha de siembra tentativa en la tercera década de junio.

Es evidente el predominio de los suelos moderadamente aptos para el cultivo de tomate en el municipio Guacara de $95,0 \mathrm{~km}^{2}$; con disponibilidad hídrica entre 396,0 y 322,0 mm en el ciclo del cultivo, con rendimientos entre 90,0 y $95,0 \%$, sembrando la primera década de junio en el centro norte y la primera década de julio para el oeste del área.

Las zonas aptas y moderadamente aptas son tierras clase I, II y III con buena fertilidad que pueden ser utilizadas para la agricultura intensiva siempre y cuando no generan problemas de compactación de suelos y quema de rastrojos. La precipitación media anual en el municipio Carlos Arvelo se encuentra entre $1.500 \mathrm{~mm}$ en el suroeste, $1.100 \mathrm{~mm}$ en el norte y valores por debajo de $900 \mathrm{~mm}$ en el sur y este del municipio. El área se sitúa entre llanuras lacustrinas, valles, colinas y montañas pertenecientes a las Regiones Naturales de la Depresión del Lago de Valencia y la Serranía del Interior (Olivares et al., 2018a).
En este sentido, se tienen aproximadamente $83,0 \mathrm{~km}^{2}$ de suelos aptos para el cultivo del tomate, que se caracterizan por texturas medianas, son moderadamente drenados y el rendimiento a obtener entre 95,0 y $100,0 \%$ sembrando en la segunda década de mayo hacia el sur (Figura 2).

Los suelos de las zonas aptas ubicadas en los municipios Bejuma, Montalbán, Guacara y San Joaquín; tienen pocas limitaciones que restringen su uso, son apropiados para el tomate incluso otras hortalizas. Son tierras en su mayoría planas, con escaso peligro de erosión. Los suelos son profundos, con buen drenaje externo e interno y fácil de trabajar. Retienen buena cantidad de agua, poseen un buen contenido de nutrientes o responden bien a los fertilizantes.

En zonas de Carabobo sin condiciones naturales optimas, se pudiera obtener un desarrollo adecuado del cultivo; indicando, que, al ubicar este cultivo en zonas agroecológicamente adecuadas, de acuerdo con sus requerimientos edáficos y climáticos, se puede llegar a un máximo potencial. En este estudio se evidenció que la variación en la fecha de siembra fue determinante en la asignación de la tierra en las distintas categorías de aptitud para el tomate.

El déficit hídrico que se manifiesta en la zona de estudio según reportes de Paredes-Trejo y Olivares (2018) afectaría negativamente el número de frutos por unidad de superficie durante el período de maduración de frutos. En la fase de crecimiento y maduración del fruto, niveles hídricos insuficientes provocan una reducción del peso unitario del fruto,

Tabla 1. Superficie $\left(\mathrm{Km}^{2}\right)$ de la aptitud del cultivo de tomate por municipio del estado Carabobo, Venezuela

\begin{tabular}{lccrr}
\hline Municipio / Superficie $\left(\mathrm{km}^{2}\right)$ & Apto (A1) & Moderadamente Apto (A2) & No Apto (NA) & Total \\
\hline Diego Ibarra & 29 & 0 & 84 & 113 \\
San Joaquín & 24 & 12 & 73 & 109 \\
Guacara & 31 & 15 & 119 & 165 \\
San Diego & 0 & 0 & 110 & 110 \\
Naguanagua & 1 & 95 & 88 & 184 \\
Los Guayos & 16 & 0 & 40 & 56 \\
Puerto Cabello & 0 & 0 & 85 & 85 \\
Juan José Mora & 0 & 0 & 295 & 295 \\
Miranda & 8 & 13 & 160 & 172 \\
Montalbán & 21 & 25 & 81 & 115 \\
Bejuma & 19 & 42 & 116 & 160 \\
Libertador & 76 & 20 & 275 & 393 \\
Valencia & 40 & 100 & 231 & 291 \\
Carlos Arvelo & 83 & 326 & 309 & 492 \\
Total & 348 & & 2066 & 2740 \\
\hline
\end{tabular}


disminuyendo por estas dos vías la productividad por superficie del cultivo (Mitchell et al., 1991).

En sectores del noroeste la fecha optima de siembra se encuentra entre la tercera década de mayo y la primera de junio; para las localidades ubicadas hacia el oeste de la localidad de Tacarigua la fecha optima de siembra se encuentra entre la primera década de Julio y la a primera del mes de septiembre; por otra parte en zonas del centro, norte y este, la fecha optima de siembra se ubica entre la tercera década de Julio y la primera de agosto, y finalmente, en áreas de llanura lacustrina del norte y en las proximidades de la localidad de Belén, la fecha más idónea seria únicamente para la primera década de agosto, estos resultados coinciden con la distribución espacial del índice hídrico desarrollado por Olivares et al. (2018b).

En $100,0 \mathrm{~km}^{2}$ del municipio Carlos Arvelo, los suelos son excesivamente drenados de texturas arenosas y se encuentran en pendientes del terreno entre 6,0 y $10,0 \%$, por lo que se clasificaron como tierras moderadamente aptas para el tomate, pudiendo obtener máximo rendimiento de $95 \%$ durante la cosecha. La fecha de siembra estimada para las áreas moderadamente aptas para el tomate es durante la segunda década de mayo hacia el sur del municipio y la segunda década de agosto únicamente en el resto de los suelos categorizados como moderadamente aptos (Figura 2).

El municipio Libertador se emplaza en el suroccidente del estado Carabobo; la mayor parte del municipio se encuentra en zonas de valles y colinas, con precipitación anual promedio entre $1.500 \mathrm{~mm}$ al sureste y $1.000 \mathrm{~mm}$ en el noroeste del municipio. Las condiciones edafoclimáticas del municipio Libertador, determinan aproximadamente $76,0 \mathrm{~km}^{2}$ de tierras aptas para el cultivo de tomate, ubicadas en las áreas bajas de las colinas y valles del municipio, mientras que en las partes altas de las colinas, se identificaron $42,0 \mathrm{~km}^{2}$ de suelos moderadamente aptos para el tomate (Figura 2), ya que la disponibilidad hídrica en estas zonas es inferior a 262,0 $\mathrm{mm}$ durante los 65 días de la fase crítica del rubro, además, de que los suelos son de texturas ligeras y excesivamente drenados, por lo que la humedad necesaria no es retenida eficientemente.

Las fechas de siembra para la mayor parte de los suelos aptos, se estimó para la segunda década de mayo, en algunos sectores del suroeste de la localidad de Tocuyito, esta comprende 20 días, desde la tercera década de mayo hasta la primera de junio. Se estima la fecha de siembra hacia el este de la localidad de Campo de Carabobo, para la primera década de Julio únicamente, mientras que, para el restante de los suelos, puede sembrarse estrictamente en la tercera década del mes de agosto.

En lo referido a los suelos moderadamente aptos, la fecha de siembra se estima para las áreas bajas de colinas durante la tercera década de mayo, mientras que, en algunas tierras del noroeste, la fecha corresponde para la primera década de junio. Hacia el este y el noroeste, se tienen tierras cuya fecha se calculó para la primera década de julio, por último, el resto de los suelos moderadamente aptos para el tomate, cuentan con fecha de siembra únicamente de la tercera década de agosto, para obtener entre 90,0 y $95,0 \%$ de rendimiento.

Las condiciones geomorfológicas del municipio Valencia del estado Carabobo, muestran que, la zona se encuentra entre paisajes de montañas, colinas y valles, entre la Depresión del Lago de Valencia, la Serranía del Interior y estribaciones del inicio de la Serranía del Litoral, en donde la precipitación media anual está entre $1.500 \mathrm{~mm}$ al sur del área y cerca de $1.100 \mathrm{~mm}$ en las montañas y valle del norte, en donde se asienta la ciudad de Valencia. Aproximadamente $40,0 \mathrm{~km}^{2}$ de los suelos del municipio son aptos para el cultivo de tomate, cuyas fechas de siembra se estimaron para la segunda década de mayo en el centro y sureste, y durante la tercera década de mayo hacia el sur de la ciudad de Valencia.

El resto de las tierras aptas para el cultivo de tomate, se estimaron con fecha de siembra entre la primera década de julio y la tercera de agosto para obtener rendimientos entre 95,0 y 100,0\%. En las zonas altas, en donde las tierras se catalogaron como moderadamente aptas en $20,0 \mathrm{~km}^{2}$, los suelos se caracterizan por texturas ligeras, son excesivamente drenados y las pendientes del terreno están entre 6,0 y 10,0\%; pudiéndose obtener entre 90,0 y $95,0 \%$ de rendimiento durante la cosecha de tomate. La fecha de siembra estimada para las tierras moderadamente aptas del municipio Valencia, es durante la primera década de Julio únicamente, ya que luego de este período de 10 días, el rendimiento a obtener sería nulo.

\section{Conclusiones}

Las evaluaciones desarrolladas en este estudio, constituyen un avance notable en materia de zonificación agroclimática del cultivo y sobre 
todo en la disponibilidad potencial de alimentos de origen interno, pues así, como permiten conocer la cantidad de tierra vinculada a la producción y tener una idea general sobre el rendimiento medio por producto en función a las fechas de inicio del periodo lluvioso, también facilitaría el cálculo de las pérdidas de área sembrada por producto y zona climática, en caso de no seguir los lineamientos de la zonificación. Adicionalmente, los resultados alcanzados son provisionales ante la falta de experiencias previas, y otras futuras que se puedan realizar, y que por tanto de trata de hipótesis alcanzadas que están por corroborarse.

Los estudios relacionados con la adaptabilidad agroclimática son una herramienta que permite establecer condiciones físico-naturales para la planificación y ordenación territorial, que lleva inmersa la búsqueda del desarrollo sustentable de la región. La información puede servir como apoyo para la licitación y estudios de los seguros de créditos agrícolas, que requieran de información edafoclimática, para el establecimiento de pólizas a futuro, y adicionalmente para los actores sociales y económicos sumergidos en la producción agrícola en el estado Carabobo.

\section{Agradecimientos}

Al apoyo financiero de la Secretaría de Seguridad Alimentaria y Desarrollo Agrario de la Gobernación del estado Carabobo, Venezuela. También, al apoyo técnico de los investigadores del Instituto Nacional de Investigaciones Agrícolas: Adriana Cortez, Juan Carlos Rey y María Fernanda Rodríguez.

\section{Literatura Citada}

Allen, R.G.; Pereira, L.S.; Raes, D.; Smith, M.

2006. Evapotranspiración del cultivo. Guías para la determinación de los requerimientos de agua de los cultivos. Estudio FAO de Riego y Drenaje 56. FAO. Roma, Italia. 323 p.

Benacchio, S.

1982. Algunas exigencias agroecológicas en 58 especies de cultivo con potencial de producción en el trópico americano. Fondo Nacional de Investigaciones Agropecuarias. Centro Nacional de Investigaciones Agropecuarias. Ecuador. 202 p.

Cortez, A.; Rodríguez, M.F.; Rey, J.C.; Ovalles, F.; González, W.; Parra, R.; Olivares, B.; Marquina, J.

2016. Variabilidad espacio temporal de la precipitación en el estado Guárico, Venezuela. Revista de la Facultad de Agronomía de LUZ, 33: 292-310.

Doorenbos, J.; Pruitt, W.O.

1975. Guidelines for predicting crop water requirements. Organización de las Naciones Unidades para la Agricultura y la Alimentación. Estudio de Riego y Drenaje No 24. FAO. Roma, Italia. 179 p.

2012 Respuesta del rendimiento de los cultivos al agua. Estudio FAO: Riego y drenaje 66. Organización de las Naciones Unidas para la Alimentación y la Agricultura. Roma, Italia. 510 p.

Franquin, P.

1983. Modelos estadísticos sobre potenciales del período de crecimiento de cultivos. Información Climática para el Desarrollo-Reviviendo la Revolución Verde. FONAIAPBID. Maracay, Venezuela. 183 p.

Hernández, R.; Pereira, Y.; Molina, J.C.; Coelho, R.; Olivares, B.; Rodríguez, K.

2017. Calendario de siembra para las zonas agrícolas del estado Carabobo en la República Bolivariana de Venezuela. Editorial Universidad Internacional de Andalucía. Sevilla, España. 247 p.
INIA.

2016. Aproximación agroecológica para el nuevo modelo de producción agrícola en Venezuela. Instituto Nacional de Investigaciones Agrícolas, Venezuela. Maracay, Venezuela. 90 p.

IANAS -Red Interamericana de Academias de Ciencias.

2017. Retos y oportunidades de la seguridad alimentaria y nutricional en las Américas. El punto de vista de las Academias de Ciencias. IANAS. México. 637 p.

Mitchell, J.P.; Shennan, C.; Grattan, S.R.; May, D.M.

1991. Tomato fruit yields and quality under water deficit and salinity. Journal of the American Society for Horticultural Science, 116: 215-221.

Nava, A.; Londoño, A.; Polston, J.E.

2013. Characterization and distribution of tomato yellow margin leaf curl virus, a begomovirus from Venezuela. Arch. Virol,158(2): 399-406.

Olivares, B.; Hernández, R.; Arias, A.; Molina, J.C.; Pereira Y. 2018d. Zonificación agroclimática del cultivo de maíz para la sostenibilidad de la producción agrícola en Carabobo, Venezuela. Revista Universitaria de Geografía, 27: 139-159. Olivares, BO.

2018. Condiciones tropicales de la lluvia estacional en la agricultura de secano de Carabobo, Venezuela. La Granja: Revista de Ciencias de la Vida, 27: 86-102.

Olivares, BO.; Cortez, A.; Parra, R.; Rodríguez, M.; Guevara, E. 2013. Aplicación de procedimientos estadísticos para el control de calidad de las series de precipitación mensual de los llanos orientales venezolanos. Revista de la Facultad de Agronomía de LUZ, 30: 367-391.

Olivares, B.O.; Hernández, R.; Arias, A.; Molina, J.C.; Pereira, Y. 2018c. Identificación de zonas agroclimáticas potenciales para producción de cebolla (Allium cepa L.) en Carabobo, Venezuela. Journal of the Selva Andina Biosphere, 6: 70-82.

Olivares, B.O.; Hernández, R.; Coelho, R.; Molina, J.C.; Pereira, Y. 2018a. Analysis of climate types: Main strategies for sustainable decisions in agricultural areas of Carabobo, Venezuela. Scientia Agropecuaria, 9: 359-369. 
Olivares, B.O.; Hernández, R.; Coelho, R.; Molina, J.C.; Pereira, Y

2018b. Análisis espacial del índice hídrico: un avance en la adopción de decisiones sostenibles en territorios agrícolas de Carabobo, Venezuela. Revista Geográfica de América Central, 60: 277-299.

Olivares, B.O.; Zingaretti, M.L.

2018. Análisis de la sequía meteorológica en cuatro localidades agrícolas de Venezuela mediante la combinación de métodos multivariados. UNED Research Journal, 10: 181-192.

Olivares, B.O.; Zingaretti, M.L.; Demey, Z.J.A.; Demey, J.R. 2016. Tipificación de los sistemas de producción agrícola y la percepción de la variabilidad climática en Anzoátegui, Venezuela. FAVE - Ciencias Agrarias, 15: 39-50.

Ortega-Farías, S.; Calderon R.; Acevedo, C.; Fuentes, S. 2000. Estimación de la evapotranspiración real diaria de un cultivo de tomates usando la ecuación de PenmamMonteith. Ciencia Investigaçao Agraria, 27(2): 91-96.
Paredes-Trejo, F.; Olivares B.

2018. El desafio de la sequía en Venezuela. En: Núñez Cobo, J.; Verbist, K. (eds.). Atlas de Sequía de América Latina y el Caribe. UNESCO. Francia. pp. 127-136.

Parra, R.; Cortez, A.

2005. Control de calidad de series de precipitación de las series de precipitación del INIA Venezuela en el periodo 1970-2000. Revista Argentina de Agrometeorología, 5-6: 63-73.

Salas, M.; Delgado, F.; Esteva,Y.; Sampson, M. 2008. La ordenación del territorio y la vocación de uso agrícola de la tierra en Venezuela. Revista Geográfica Venezolana, 49: 267-288.

Solís, A.; Martínez, R.; Moya, C.; Dominí, M.E.; López, V.; Milán, E.; Amat, I.

2006. Comportamiento de variedades de tomate (Lycopersicon esculentum Mill) en dos períodos de siembra en la localidad de Velasco, provincia Holguín. Cultivos Tropicales, 27: 51-54.

Villa, M.M.; Inzunza, M.A.; Catalán, E.A.

2001. Zonificación agroecológica de hortalizas involucrando grados de riesgo. Terra, 19: 1-7. 